\title{
ON THE CENTERS OF CUBIC POLYNOMIAL DIFFERENTIAL SYSTEMS WITH FOUR INVARIANT STRAIGHT LINES
}

\author{
JAUME LLIBRE
}

\begin{abstract}
Assume that a cubic polynomial differential system in the plane has four invariant straight lines in generic position, i.e. they are not parallel and no more than two straight lines intersect in a point. Then such a differential system only can have 0,1 or 3 centers.
\end{abstract}

\section{INTRODUCTION AND STATEMENT OF THE MAIN RESULTS}

A center of a differential system in $\mathbb{R}^{2}$ is an equilibrium point $p$ for which there exists a neighborhood $U$ of $p$ such that $U \backslash\{p\}$ is filled by periodic orbits. The equilibrium point $p$ is a focus if there exists a neighborhood $U$ of $p$ where all the orbits in $U \backslash\{p\}$ spiral tending to $p$ either in backward, or in forward time. These definitions of focus and center goes back to Dulac [10] and Poincaré [23].

The problem of distinguish between a focus or a center (known as the centerfocus problem) is a classical problem in the qualitative theory of planar polynomial differential systems, which is related to the Hilbert 16th problem, see Hilbert [14], Ilyashenko [15], Li [19].

For the polynomial differential systems of degree 2 the center-focus problem was solved by Bautin [3], Kapteyn [16, 17], Schlomiuk [24], Vulpe [25], Żołạdek [28]. However, this problem remains unsolved for polynomial differential systems of degree 3 , simply called from now on cubic systems.

The centers of many different subclasses of cubic differential systems have been studied. Thus reversible cubic systems with a center has been classified by Żoładek $[30,31]$, and Buzzi et al [4]. the centers of cubic systems without quadratic terms also were classified by Malkin [21], Vulpe and Sibirskii [26], Żołądek [29], ... The classification of the Hamiltonian linear type centers and the nilpotent ones for cubic systems without quadratic terms has been recently classified by Colak et al $[5,6,7,8]$. The centers of the Kolmogorov cubic systems have been analyzed and classified in $[20]$.

In this work we study the centers for the cubic systems having four invariant straight lines in generic position, i.e. they are not parallel and no more than two straight lines intersect in a point. Our main result is the following.

2010 Mathematics Subject Classification. 37K10, 37C27, 37K05.

Key words and phrases. cubic system, cubic polynomial differential systems, centers, invariant straight line. 
Theorem 1. Cubic systems having four invariant straight lines in generic position only can have 0,1 or 3 centers.

Theorem 1 is proved in section 3 .

In section 2 we summarize some well known results that we shall use for proving Theorem 1.

\section{Preliminary Results}

2.1. Equilibrium points. We say that an equilibrium point $p$ of a differential system in $\mathbb{R}^{2}$ is elementary if the determinant of the linear part of the system at $p$ is non-zero.

An elementary equilibrium $p$ is hyperbolic if the two eigenvalues of the linear part of the system at $p$ have non-zero real part. The elementary equilibria only can be saddles with topological index -1 and the corresponding determinant negative; nodes or foci, these last two with topological index 1 and the corresponding determinant positive. We recall that the trace of the matrix of the linear part of a planar differential system at an elementary node is not zero. For a proof and definitions of saddle, node and focus and their properties here mentioned see for instance Chapter 2 of [11].

It is known that an elementary equilibrium point of an analytic differential system in $\mathbb{R}^{2}$ which is not hyperbolic, has eigenvalues purely imaginary, and only can be a focus or a center, for more details see [1].

2.2. Poincaré compactification. Let $\mathbb{S}^{2}=\left\{y \in \mathbb{R}^{3}:\|y\|=1\right\}$ be the $2-$ dimensional sphere, here $\|\cdot\|$ denotes the Euclidean norm in $\mathbb{R}^{3}$. The Poincaré compactification of a polynomial vector field $\mathcal{X}$, denoted by $p(\mathcal{X})$, is an induced vector field on the sphere $\mathbb{S}^{2}$ defined as follows. For additional details on the Poincaré compactification see Chapter 5 of [11].

Let $T_{y} \mathbb{S}^{2}$ be the tangent space to $\mathbb{S}^{2}$ at the point $y$. Consider $\mathcal{X}$ defined in the plane $T_{(0,0,1)} \mathbb{S}^{2}$ identified with $\mathbb{R}^{2}$. The central projection $f: T_{(0,0,1)} \mathbb{S}^{2} \rightarrow \mathbb{S}^{2}$ sends every point $y$ of the plane $T_{(0,0,1)} \mathbb{S}^{2}$ to two points on $\mathbb{S}^{2}$, the ones which are in the intersection of $\mathbb{S}^{2}$ with the straight line passing through the point $y$ and the center of the sphere $\mathbb{S}^{2}$, i.e. the origin of coordinates. This map defines two copies of $\mathcal{X}$, one in the open northern hemisphere $\mathbb{H}^{+}$and other in the open southern hemisphere $\mathbb{H}^{-}$. Denote by $\mathcal{X}^{\prime}$ the vector field $D f \circ \mathcal{X}$ defined on $\mathbb{S}^{2}$ except on its equator $\mathbb{S}^{1}=\left\{y=\left(y_{1}, y_{2}, y_{3}\right) \in \mathbb{S}^{2}: y_{3}=0\right\}$. Clearly the infinity of $\mathbb{R}^{2} \equiv T_{(0,0,1)} \mathbb{S}^{2}$ is identified with the equator $\mathbb{S}^{1}$ of $\mathbb{S}^{2}$. The polynomial vector field $\mathcal{X}^{\prime}$ of degree $n$ can be extended to an analytic vector field $p(\mathcal{X})$ on $\mathbb{S}^{2}$ taking $p(\mathcal{X})=y_{3}^{n-1} \mathcal{X}^{\prime}$. On $\mathbb{S}^{2} \backslash \mathbb{S}^{1}=\mathbb{H}^{+} \cup \mathbb{H}^{-}$there are two copies of $\mathcal{X}$, one in $\mathbb{H}^{+}$and the other in $\mathbb{H}^{-}$, and knowing the behaviour of $p(\mathcal{X})$ around $\mathbb{S}^{1}$, we know the behaviour of $\mathcal{X}$ at infinity. The Poincaré compactification has the property that $\mathbb{S}^{1}$ is invariant under the flow of $p(\mathcal{X})$.

The finite equilibrium points of $\mathcal{X}$ or of $p(\mathcal{X})$ are the equilibrium points of $\mathcal{X}$, while the equilibrium points of $p(\mathcal{X})$ contained in $\mathbb{S}^{1}$, i.e. at infinity, are called the 
infinite equilibrium points of $\mathcal{X}$ or of $p(\mathcal{X})$. We note that the infinity equilibrium points appear in pairs diametrically opposed.

Let $\mathbb{R}[x, y]$ be the ring of real polynomials in the variables $x$ and $y$. A planar polynomial differential system of degree $m$ is a differential system

$$
\dot{x}=P(x, y), \quad \dot{y}=Q(x, y),
$$

with $P, Q \in \mathbb{R}[x, y]$ satisfying $\max \{\operatorname{deg} P, \operatorname{deg} Q\}=m$. As usual the dot denotes derivative with respect to an independent variable $t$, usually called the time. We denote by

$$
\mathcal{X}=P \frac{\partial}{\partial x}+Q \frac{\partial}{\partial y}
$$

the polynomial vector field associated to system (1).

For working with the vector field $p(\mathcal{X})$ on the sphere $\mathbb{S}^{2}$ we use the six local charts given by $U_{k}=\left\{y \in \mathbb{S}^{2}: y_{k}>0\right\}, V_{k}=\left\{y \in \mathbb{S}^{2}: y_{k}<0\right\}$ for $k=1,2,3$. The corresponding local maps $\phi_{k}: U_{k} \rightarrow \mathbb{R}^{2}$ and $\psi_{k}: V_{k} \rightarrow \mathbb{R}^{2}$ are defined as $\phi_{k}(y)=-\psi_{k}(y)=\left(y_{m} / y_{k}, y_{n} / y_{k}\right)$ for $m<n$ and $m, n \neq k$. We denote by $z=(u, v)$ the value of $\phi_{k}(y)$ or $\psi_{k}(y)$ for any $k$, so the coordinates $(u, v)$ will play different roles depending on the local chart we are considering. The points in the infinity $\mathbb{S}^{1}$ in any chart have the coordinate $v=0$.

The expression for $p(\mathcal{X})$ in local chart $\left(U_{1}, \phi_{1}\right)$ is given by

$$
\begin{aligned}
\dot{u} & =v^{m}\left[-u P\left(\frac{1}{v}, \frac{u}{v}\right)+Q\left(\frac{1}{v}, \frac{u}{v}\right)\right], \\
\dot{v} & =-v^{m+1} P\left(\frac{1}{v}, \frac{u}{v}\right) .
\end{aligned}
$$

The expression for $\left(U_{2}, \phi_{2}\right)$ is

$$
\begin{aligned}
& \dot{u}=v^{m}\left[P\left(\frac{u}{v}, \frac{1}{v}\right)-u Q\left(\frac{u}{v}, \frac{1}{v}\right)\right], \\
& \dot{v}=-v^{m+1} Q\left(\frac{u}{v}, \frac{1}{v}\right),
\end{aligned}
$$

and for $\left(U_{3}, \phi_{3}\right)$ is

$$
\begin{aligned}
& \dot{u}=P(u, v), \\
& \dot{v}=Q(u, v) .
\end{aligned}
$$

The expression for $p(\mathcal{X})$ in the charts $\left(V_{k}, \psi_{k}\right)$ is the same as for $\left(U_{k}, \phi_{k}\right)$ multiplied by $(-1)^{d-1}$, for $k=1,2,3$.

2.3. Poincaré-Hopf Theorem. The next result is the Poincaré-Hopf Theorem for a vector field defined on the 2-dimensional sphere $\mathbb{S}^{2}$, see an elementary proof in Chapter 6 of [11].

Theorem 2. For every tangent vector field on the sphere $\mathbb{S}^{2}$ with a finite number of equilibria, the sum of their topological indices is 2 . 
2.4. Arrangement of four straight lines. We consider an arbitrary arrangement of four straight lines in generic position. Then doing an affine change of variables we can assume that these four straight lines are $x=0, y=0, x+a y+b=0$ and $x+c y+d=0$, satisfying that

$$
a b c d(a-c)(b-d)(b c-a d) \neq 0 .
$$

For more information on arrangements of straight lines see for instance [22].

2.5. Normal forms of polynomial differential systems having a set of given generic invariant algebraic curves. The algebraic curve $C(x, y)=0$ of $\mathbb{R}^{2}$ is called an invariant algebraic curve of system (1) if

$$
P C_{x}+Q C_{y}=K C,
$$

for some real polynomial $K(x, y)$. With this definition it is easy to check that the algebraic curve $C=0$ is formed by orbits of system (1).

Theorem 3. Let $C_{i}=0$ for $i=1, \cdots, p$ be irreducible invariant algebraic curves in $\mathbb{R}^{2}$, and let $r=\sum_{i=1}^{p} \operatorname{degree}\left(C_{i}\right)$. We assume that all $C_{i}$ satisfy the following generic conditions:

(i) There are no points at which $C_{i}$ and its first derivatives are all vanish.

(ii) The highest order terms of $C_{i}$ have no repeated factors.

(iii) If two curves intersect at a point in the finite plane, they are transversal at this point.

(iv) There are no more than two curves $C_{i}=0$ meeting at any point in the finite plane.

(v) There are no two curves having a common factor in the highest order terms.

If $r=m+1$ then any polynomial vector field $\mathcal{X}$ of degree $m$ tangent to all $C_{i}=0$ is of the form

$$
\mathcal{X}=\sum_{i=1}^{p} \alpha_{i}\left(\prod_{\substack{j=1 \\ j \neq i}}^{p} C_{j}\right) \mathcal{X}_{C_{i}},
$$

with $\alpha_{i} \in \mathbb{R}$.

Theorem 2 is proved in [9], where also it is explained the history of this result.

Let $U$ be an open subset of $\mathbb{R}^{2}$. A non-locally constant function $H: U \rightarrow \mathbb{R}$ is a first integral of system (1) in $U$ if it is constant on all the orbits of system (1) contained in $U$, i.e.

in all the points of $U$.

$$
\dot{H}=\mathcal{X} H=P \frac{\partial H}{\partial x}+Q \frac{\partial H}{\partial y}=0,
$$

A function $R: U \rightarrow \mathbb{R}$ not identically zero is an integrating factor of system (1) if it satisfies

$$
\frac{\partial(R P)}{\partial x}+\frac{\partial(R Q)}{\partial y}=0
$$


A first integral $H$ associated to this integrating factor $R$ is

$$
H(x, y)=-\int R(x, y) P(x, y) d y+f(x),
$$

where $H(x, y)$ must satisfy $\partial H / \partial x=R Q$.

Theorem 4. Under the assumptions of Theorem 3 the polynomial vector field (5) has the integrating factor $1 /\left(C_{1} \cdots C_{p}\right)$.

For a proof see the paper of Christopher and Kooij [18].

2.6. Bezout Theorem. For a proof of the next result known as the Bezout Theorem see for instance [12].

Theorem 5. Let $R(x, y)$ and $S(x, y)$ be two real polynomials. If both polynomials do not share a non-trivial common factor, then the algebraic system of equations

$$
R(x, y)=S(x, y)=0
$$

has at most degree $(R)$ degree $(S)$ solutions in $\mathbb{R}^{2}$.

We recall that a simple equilibrium $\left(x_{0}, y_{0}\right)$ of system $(1)$ is an equilibrium satisfying

$$
\operatorname{det}\left(\frac{\partial(P, Q)}{\partial(x, y)}\left(x_{0}, y_{0}\right)\right) \neq 0 \text {. }
$$

If a polynomial differential system (1) of degree $m$ has its maximum number of equilibria $m^{2}$, then all its equilibria are simple, and consequently elementary. See for a proof [2] or [13].

\section{Proof of Theorem 1}

We consider the four straight lines in generic position described in subsection 2.4. Applying Theorem 3 we obtain all cubic systems having the mentioned four straight lines as invariant algebraic curves, which are

$$
\begin{aligned}
\dot{x}= & -x\left(b d \beta+(b+d) \beta x+(a d(\beta+\gamma)+b c(\beta+\delta)) y+\beta x^{2}\right. \\
& \left.+(a(\beta+\gamma)+c(\beta+\delta)) x y+a c(\beta+\gamma+\delta) y^{2}\right), \\
\dot{y}= & y\left(b d \alpha+(b(\alpha+\delta)+d(\alpha+\gamma)) x+(a d+b c) \alpha y+(\alpha+\gamma+\delta) x^{2}\right. \\
& \left.+(a(\alpha+\delta)+c(\alpha+\gamma)) x y+a \alpha c y^{2}\right) .
\end{aligned}
$$

By Theorem 4 we know that the function

$$
R(x, y)=\frac{1}{x y(x+a y+b)(x+c y+d)}
$$

is an integrating factor of system (6), and from subsection 2.5 the associated first integral to this integrating factor is

$$
H(x, y)=x^{\alpha} y^{\beta}(x+a y+b)^{\gamma}(x+c y+d)^{\delta} .
$$

Claim 1: If some of parameters $\alpha, \beta, \gamma$ and $\delta$ is zero, then the cubic system (6) has at most one center. 
Proof of Claim 1. We assume that $\alpha=0$, the proof if $\beta, \gamma$ or $\delta$ is zero is exactly the same. Then the equilibria of system (6) are all the points of the straight line $x=0$ and the points

$$
\begin{gathered}
(-b, 0),(-d, 0),\left(\frac{b c-a d}{a-c}, \frac{d-b}{a-c}\right) \\
\left(\frac{b(c(\beta+\delta)-a \delta)-d(a(\beta+\gamma)-c \gamma)}{(a-c)(\beta+\gamma+\delta)}, \frac{\beta(d-b)}{(a-c)(\beta+\gamma+\delta)} .\right) .
\end{gathered}
$$

Since the unique equilibrium point which is not on an invariant straight line is the last one if exists, i.e. if $\beta+\gamma+\delta \neq 0$, it is clear that when $\alpha=0$ at most we can have one center.

From Claim 1 in what follows we can assume that

$$
\alpha \beta \gamma \delta \neq 0 \text {. }
$$

Since the four invariant straight lines are in generic position they intersect in six equilibrium points, namely

$$
(0,0),(-b, 0),(-d, 0),\left(0,-\frac{b}{a}\right),\left(0,-\frac{d}{c}\right),\left(\frac{b c-a d}{a-c}, \frac{d-b}{a-c}\right) .
$$

Note that from (4) all these equilibria exist. The determinant of the linear part of system (6) in these equilibria are

$$
\begin{gathered}
-b^{2} d^{2} \alpha \beta,-b^{2}(b-d)^{2} \beta \gamma,-(b-d)^{2} d^{2} \beta \delta,-\frac{b^{2}(b c-a d)^{2}}{a^{2}} \alpha \gamma \\
-\frac{d^{2}(b c-a d)^{2}}{c^{2}} \alpha \delta,-\frac{(b-d)^{2}(b c-a d)^{2}}{(a-c)^{2}} \gamma \delta
\end{gathered}
$$

respectively. From (4) and (8) all these determinants never vanish, consequently all the equilibria (9) are elementary. Therefore, by subsection 2.1 they only can be saddles, nodes, focus or centers, but since they are on invariant straight lines they are either saddles or nodes. Saddles when their corresponding determinant is negative, and nodes when they are positive.

On the other hand by Bezout Theorem (see Theorem 5) the cubic system (6) has at most nine equilibria, since six of them are on invariant straight lines it follows that system (6) has at most three centers.

Claim 2: The trace of the matrix of the linear part of the cubic system (6) at an equilibrium point which is not on the invariant straight lines is zero, or system (6) has at most one center.

Proof of Claim 2. The equilibria which are not on the invariant straight lines are solution of the system $\dot{x} / x=\dot{y} / y=0$, with the exception of the equilibrium point $((b c-a d) /(a-c),(d-b) /(a-c))$. We isolate $x^{2}$ from the equation $\dot{x} / x=0$ and substitute it into the equation $\dot{y} / y=0$. Now from this last equation we isolate $x$ 
and we obtain $x=-p_{1}(y) / p_{2}(y)$ assuming that $p_{2}(y) \neq 0$, where

$$
\begin{aligned}
p_{1}(y)= & b c(\alpha \delta+(\beta+\delta)(\gamma+\delta)))+(a d(\alpha \gamma+(\beta+\gamma)(\gamma+\delta)) y \\
& +a c(\gamma+\delta)(\alpha+\beta+\gamma+\delta)+b \beta d(\gamma+\delta) y^{2} \\
p_{2}(y)= & \beta(b \gamma+d \delta)+(\alpha+\beta+\gamma+\delta)(a \gamma+c \delta) y .
\end{aligned}
$$

Later on we shall study the case $p_{2}(y)=0$.

Substituting $x=-p_{1}(y) / p_{2}(y)$ into the equation $\dot{x} / x=0$ we get the following equation only in the variable $y$ :

$$
\frac{\beta \gamma \delta(b-d+(a-c) y) p_{3}(y)}{p_{2}(y)^{2}}=0,
$$

where

$$
\begin{aligned}
p_{3}(y)= & b \beta^{2} d(b-d)+\beta(b d(a(\alpha+2(\beta+\gamma)+\delta)-c(\alpha+2 \beta+\gamma+2 \delta)) \\
& \left.-a d^{2}(\alpha+\beta+\gamma)+b^{2} c(\alpha+\beta+\delta)\right) y+(\alpha+\beta+\gamma+\delta) \\
& \left(a^{2} d(\beta+\gamma)+a c(b(\alpha+2 \beta+\delta)-d(\alpha+2 \beta+\gamma))-b c^{2}(\beta+\delta)\right) y^{2} \\
& +a c(a-c)(\alpha+\beta+\gamma+\delta)^{2} y^{3} .
\end{aligned}
$$

The real roots of the polynomial $p_{3}(y)$ provide the coordinates $y$ of the equilibria which are not on the invariant straight lines, and substituting these roots into $x=-p_{1}(y) / p_{2}(y)$ we obtain the coordinate $x$ of these equilibria.

Substituting $x=-p_{1}(y) / p_{2}(y)$ into the divergence of the cubic system (6), i.e. into $\partial \dot{x} / \partial x+\partial \dot{y} / \partial y$ we obtain

$$
\text { divergence }=\frac{p_{3}(y) p_{4}(y)}{p_{2}(y)^{2}},
$$

where

$$
\begin{aligned}
p_{4}(y)= & b \gamma(\beta(\gamma+2 \delta)-\delta(\alpha+\gamma+\delta))+d \delta(\gamma(\alpha-2 \beta+\gamma)+\delta(\gamma-\beta)) \\
& +(a \gamma(\alpha(\gamma-2 \delta)+\beta(\gamma+2 \delta)+(\gamma+\delta)(\gamma-2 \delta))-c \delta(\delta(\alpha+\beta-\gamma) \\
& \left.\left.-2 \gamma(\alpha-\beta+\gamma)+\delta^{2}\right)\right) y
\end{aligned}
$$

Since the real roots of the polynomial $p_{3}(y)$ provide the $y$ coordinate of the equilibria which are not on the invariant straight lines, and this polynomial is a factor of the divergence of the system, it follows that the linear part of system (6) at all the equilibria which are not on the invariant straight lines is zero when the polynomial $p_{2}(y)$ is not the zero polynomial.

Now assume that $p_{2}(y)=0$, looking at the two coefficients of this polynomial we consider four cases.

Case 1: $\beta=0$ and $\delta=-\alpha-\gamma$. In this case it is easy to check that all the equilibrium points of system (6) are on the invariant straight lines, consequently this system has no centers. 
Case 2: $\beta=0$ and $\gamma=-c \delta / a$. Now the unique equilibrium point of system (6) which is not on the invariant straight lines is

$$
\left(\frac{a(b c-a d) \alpha}{(a-c)(a(\alpha+\delta)-c \delta)}, \frac{d-b}{a-c}\right) .
$$

Consequently system (6) has at most one center.

Case 3: $\gamma=d(\alpha+\beta) /(b-d)$ and $\delta=b(\alpha+\beta) /(d-b)$. As in Case 1 in this case system (6) has no equilibria outside the invariant straight lines, so no centers.

Case 4: $\gamma=-c \delta / a=-d \delta / b$. This case is not possible because would imply that $a d-b c=0$, in contradiction with (4).

This completes the proof of this claim.

In order to complete the proof of Theorem 1 we need to prove the following four claims.

Claim 3: Provide an example of cubic system (6) having exactly three center.

Claim 4: Provide an example of cubic system (6) having exactly one center.

Claim 5: Provide an example of cubic system (6) without centers.

Claim 6: Show that the cubic systems (6) cannot have only two centers.

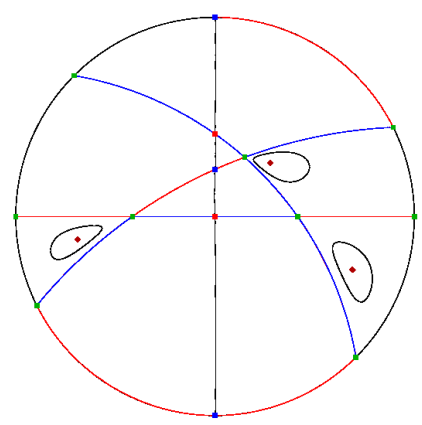

Figure 1. Phase portrait in the Poincaré disc of the cubic system (12).

Proof of Claim 3. Take $a=d=\beta=\gamma=\delta=1, b=-1, c=-2$ and $\alpha=-4$, then system (6) becomes

$$
\begin{aligned}
& \dot{x}=x\left(1-6 y-x^{2}+2 x y+6 y^{2}\right), \\
& \dot{y}=y\left(4-12 y-2 x^{2}+3 x y+8 y^{2}\right) .
\end{aligned}
$$

This system has three equilibria which are not on the invariant straight lines, namely

$$
\left(x_{1}, y_{1}\right), \quad\left(x_{2}, y_{2}\right), \quad\left(x_{3}, y_{3}\right)
$$

where

$x_{1}$ is the root 0.648190032939388 . of the polynomial $p(x)=16-24 x-3 x^{2}+3 x^{3}$, and $y_{1}$ is the root 0.630709938165919 .. of the polynomial $q(y)=-2-3 y+6 y^{2}+6 y^{3}$; $x_{2}$ is the root 3.04974949521986 .. of the polynomial $p(x)$, and $y_{2}$ is the root -1.184541590375086 .. of the polynomial $q(y)$; 
$x_{3}$ is the root -2.697939528159256 . of the polynomial $p(x)$, and $y_{3}$ is the root -0.446168347790832 .. of the polynomial $q(y)$.

We note that there are explicit exact expressions for the roots of a cubic polynomial, but we prefer present these roots in this shorter way.

The determinants of the matrices of the linear part of system (12) at the equilibrium points $\left(x_{k}, y_{k}\right)$ for $k=1,2,3$ are:

For $k=1$ is $0.8200842208962 .$. , a root of the polynomial $r(\lambda)=-4800+6000 \lambda-$ $180 \lambda^{2}+\lambda^{3}$

For $k=2$ is $136.2086577457645 .$. , a root of the polynomial $r(\lambda)$;

For $k=3$ is $42.9712580333392 .$. , a root of the polynomial $r(\lambda)$.

Since the three determinants at the equilibrium point $\left(x_{k}, y_{k}\right)$ for $k=1,2,3$ are positive, by subsection 2.1 these equilibria only can be nodes, foci or centers. They cannot be foci because in a neighborhood of them it is defined the first integral (7), also they cannot be nodes because the the trace of the matrix of the linear part of a differential system at a node is not zero, but we know by the Claim 2 that the trace of every equilibrium point which is not on the invariant straight lines is zero. In short these three equilibria are centers. See the phase portrait in the Poincaré disc of the cubic system (12) in Figure 1.

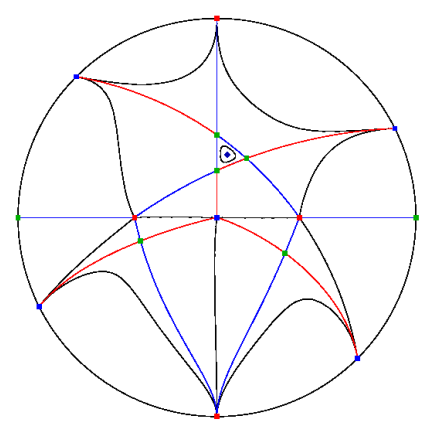

Figure 2. Phase portrait in the Poincar'e disc of the cubic system (13).

Proof of Claim 4. Take $a=d=\alpha=\beta=\gamma=\delta=1, b=-1$ and $c=-2$, then system (6) becomes

$$
\begin{aligned}
& \dot{x}=x\left(1-6 y-x^{2}+2 x y+6 y^{2}\right), \\
& \dot{y}=y\left(1-3 y-3 x^{2}+2 x y+2 y^{2}\right) .
\end{aligned}
$$

This system has three equilibria which are not on the invariant straight lines, namely

$$
\left(x_{1}, y_{1}\right), \quad\left(x_{2}, y_{2}\right), \quad\left(x_{3}, y_{3}\right),
$$

where

$x_{1}$ is the root 0.11303685689460 .. of the polynomial $p(x)=1-9 x+12 x^{3}$, and $y_{1}$ is the root 0.702574789251432 .. of the polynomial $q(y)=-2-9 y+24 y^{3}$; $x_{2}$ is the root -0.916993298352020 .. of the polynomial $p(x)$, and $y_{2}$ is the root -0.282066739245770 .. of the polynomial $q(y)$; 
$x_{3}$ is the root 0.803956441457416 . of the polynomial $p(x)$, and $y_{3}$ is the root -0.420508050005661 .. of the polynomial $q(y)$.

The determinants of the matrices of the linear part of system (12) at the equilibrium points $\left(x_{k}, y_{k}\right)$ for $k=1,2,3$ are:

For $k=1$ is $0.1615865516314450 .$. , a root of the polynomial $r(\lambda)=-108+621 \lambda+$ $288 \lambda^{2}+32 \lambda^{3}$

For $k=2$ is $-4.269300326605967 . .$, a root of the polynomial $r(\lambda)$;

For $k=3$ is $-4.89228622502547 .$. , a root of the polynomial $r(\lambda)$.

As in the proof of Claim 3 the equilibrium point having positive determinant is a center, and from subsection 2.1 the two equilibrium points with negative determinant are saddles. See the phase portrait in the Poincaré disc of the cubic system (13) in Figure 2.

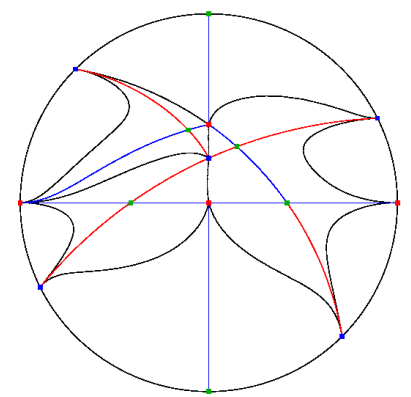

Figure 3. Phase portrait in the Poincar'e disc of the cubic system (14).

Proof of Claim 5. Take $a=d=\beta=\gamma=\delta=1, b=-1, c=-2$ and $\alpha=-1$, then system (6) becomes

$$
\begin{aligned}
& \dot{x}=x\left(1-6 y-x^{2}+2 x y+6 y^{2}\right), \\
& \dot{y}=y\left(1+x^{2}-3 y+2 y^{2}\right) .
\end{aligned}
$$

This system has only one equilibrium point which is not on the invariant straight lines, namely

$$
\left(x_{1}, y_{1}\right)=(-0.2616677544145299 . ., 0.918121959154146 . .),
$$

where $x_{1}$ is a of the polynomial $1+3 x+12 x^{3}$, and $y_{1}$ is a root of the polynomial $-2+15 x-36 x^{2}+24 x^{3}$.

The determinant of the matrix of the linear part of system (14) at the equilibrium point $\left(x_{1}, y_{1}\right)$ is -0.946250815810897 .., a root of the polynomial $36+117 \lambda+144 \lambda^{2}+$ $64 \lambda^{3}$. Since this determinant is negative the equilibrium point $\left(x_{1}, y_{1}\right)$ is a saddle. Hence system (14) has no centers. See the phase portrait in the Poincaré disc of the cubic system (14) in Figure 3.

Now we shall study the equilibrium points of system (6) at infinity using the Poincaré compactification described in subsection 2.2. Thus system (6) in the local 
chart $U_{1}$ has the expression

$$
\begin{aligned}
\dot{u}= & (\alpha+\beta+\gamma+\delta)\left(u-u^{2}-2 u^{3}\right)+(\gamma-\delta) u v+(3 \alpha+3 \beta+\gamma+2 \delta) u^{2} v \\
& -(\alpha+\beta) u v^{2} \\
\dot{v}= & \beta v-(\beta-\gamma+2 \delta) u v-\beta v^{3}-2(\beta+\gamma+\delta) u^{2} v+(3 \beta+\gamma+2 \delta) u v^{2} .
\end{aligned}
$$

In this local chart the system has three equilibrium at infinity $(0,0),(-1 / a, 0)$ and $(-1 / c, 0)$. The determinants of the matrices of the linear part of system (15) at these equilibria are

(16) $\beta(\alpha+\beta+\gamma+\delta),\left((a-c)^{2} \gamma(\alpha+\beta+\gamma+\delta)\right) / a^{2}$ and $\left((a-c)^{2} \delta(\alpha+\beta+\gamma+\delta)\right) / c^{2}$, respectively.

System (6) in the local chart $U_{2}$ has the expression

$$
\begin{aligned}
\dot{u}= & (\alpha+\beta+\gamma+\delta)\left(2 u+u^{2}-u^{3}\right)-(3 \alpha+3 \beta+\gamma+2 \delta) u v \\
& +(\delta-\gamma) u^{2} v+(\alpha+\beta) u v^{2}, \\
\dot{v}= & \alpha\left(2 v-3 v^{2}+v^{3}\right)+(\alpha+2 \gamma-\delta) u v-(\alpha+\beta+\gamma+\delta) u^{2} v \\
& +(\delta-\gamma) u v^{2} .
\end{aligned}
$$

The unique infinite equilibrium point of system (17) which is not already in the chart $U_{1}$ is the origin, and the determinant of the matrix of the linear part of system (17) at the origin is

$$
a^{2} c^{2} \alpha(\alpha+\beta+\gamma+\delta)
$$

Proof of Claim 6. We have that $\alpha \beta \delta \gamma \neq 0$, see (8). Then the six equilibria which are in the intersection of the four invariant straight lines are elementary because the determinants of the matrices of the linear part of system (6) at them given in (10) are non-zero, so their topological indices are -1 or 1 . From the paragraph previous to this proof the infinite equilibria are also non-elementary again because the corresponding determinants are non-zero, and again their topological indices are -1 or 1 .

From the proof of Claim 2 we know that at most we have three additional finite equilibria which are not contained on the invariant straight line. We denote by $I$ the sum of the topological indices of these finite equilibria outside the invariant straight lines, of course we take $I=0$ if such equilibria do not exist. Now we shall study the possible values of $I$.

We shall prove that the unique possible values for $I$ are $-1,0$ or 3 . Note that these values of $I$ prevents the existence of only two centers. Indeed, if $I=-1$ and we have only two centers these contribute to $I$ with a 2 (because the topological index of a center is 1 ). Since at most there is one additional finite equilibria outside the invariant straight lines, and if exists its index is -1 or 1 (because the cubic system reaches the maximal number of finite equilibria, nine, and then all the equilibria are elementary, see subsection 2.6), so adding this 1 or -1 to 2 never we can reach -1 . On the other hand if this third equilibrium outside the invariant straight lines, the two centers cannot produce that $I=1$.

If $I=3$ and the system has the two centers outside the invariant straight lines, then must be a third equilibrium outside the invariant straight lines with index 1 , and by the Claim 2 this third equilibrium is a center, because its trace is zero and 
since they are elementary (because the cubic system has nine finite equilibria) with index one only can be nodes, foci or centers, but the elementary nodes and foci have trace non-zero.

Now we shall prove that the unique possible values for $I$ are -1 or 3 . Recall that $\alpha \beta \delta \gamma \neq 0$. Taking into account only the signs of $\alpha, \beta, \gamma$ and $\delta$ we need only to consider the following three cases because changing the sign of the independent variable of system (6), i.e. changing the sign of the time, we get all the other possibilities and we do not change the topological indices of the equilibria.

Case 1: All the signs of $\alpha, \beta, \gamma$ and $\delta$ are positive. Then, from (10) the sum of the topological indices of the six equilibria which are in the intersection of the invariant straight lines is -6 , while the sum of the eight infinite equilibria (recall that these equilibria appears in pairs diametrally opposite in $\mathbb{S}^{1}$ ) is 8 , see (16) and (18). Therefore from the Poincaré-Hopf Theorem and taking into account that on the sphere $\mathbb{S}^{2}$ we have two copies of the finite equilibria we obtain $-12+2 I+8=2$. Hence $I=3$.

Case 2: Three of the signs of $\alpha, \beta, \gamma$ and $\delta$ are positive, and the other is negative. We distinguish three subcases.

Subcase 2.1: $\alpha+\beta+\gamma+\delta>0$. Now from (10) the sum of the topological indices of the six equilibria which are in the intersection of the invariant straight lines is 0 , and from (16) and (18) the sum of the eight infinite equilibria is 4 . So from the Poincaré-Hopf Theorem we have $0+2 I+4=2$, and consequently $I=-1$.

Subcase 2.2: $\alpha+\beta+\gamma+\delta=0$. In this case the polynomial $p_{3}(y)$ given in the proof of Claim 2, whose real roots are the $y$ coordinate of the equilibria which are outside the invariant straight lines, at most has one real root and if it exists is

$$
\frac{b d(d-b) \beta}{a d(b(\beta+\gamma)+d \delta)-b c(b \gamma+d(\beta+\delta))} .
$$

So in this case at most one center.

Subcase 2.3: $\alpha+\beta+\gamma+\delta<0$. Again from (10) the sum of the topological indices of the six equilibria which are in the intersection of the invariant straight lines is 0 , and from (16) and (18) the sum of the eight infinite equilibria is -4 . Therefore by the Poincaré-Hopf Theorem we have $0+2 I-4=2$, and $I=3$.

Case 3: Two of the signs of $\alpha, \beta, \gamma$ and $\delta$ are positive, and the other two negative. Here we only consider that $\alpha+\beta+\gamma+\delta \neq 0$, because the case $\alpha+\beta+\gamma+\delta=0$ follows as in the subcase 2.2. From (10) the sum of the topological indices of the six equilibria which are in the intersection of the invariant straight lines is 2 , and from (16) and (18) the sum of the eight infinite equilibria is 0 . Therefore by the Poincaré-Hopf Theorem we have $4+2 I+0=2$, and $I=-1$.

This completes the proof of the claim.

\section{ACKNOWLEDGEMENTS}

The first author is partially supported by the MINECO grants MTM2013-40998$\mathrm{P}$ and MTM2016-77278-P (FEDER), the AGAUR grant 2014 SGR568, and the projecte MDM-2014-0445 (BGSMath). 


\section{REFERENCES}

[1] A.A. Andronov, E.A. Leontovich, I.I. Gordon, And A.G. Maier, Qualitative theory of second-order dynamic systems, Israel Program for Scientific Translations. Halsted Press (A division of Wiley), New York, 1973.

[2] V.I. Arnold, A. VArchenko And S. Goussein-Zude, Singularits des applications différentiables, Mir, Moscou, 1982.

[3] N.N. BAUTIN, On the number of limit cycles which appear with the variation of coefficients from an equilibrium position of focus or center type, Mat. Sbornik 30 (1952), 181-196, Amer. Math. Soc. Transl. Vol. 100 (1954), 1-19.

[4] C.A. Buzzi, J. Llibre And J.C. Medrado, Phase portraits of reversible linear differential systems with cubic homogeneous polynomial nonlinearities having a non-degenerate center at the Origin, Qual. Theory Dyn. Syst. 7 (2009), 369-403.

[5] I.E. COLAK, J. Llibre AND C. VAlls, Hamiltonian linear type centers of linear plus cubic homogeneous polynomial vector fields, J. Differential Equations 257 (2014), 1623-1661.

[6] I.E. Colak, J. Llibre AND C. VAlls, Hamiltonian nilpotent centers of linear plus cubic homogeneous polynomial vector fields, Advances in Mathematics 259 (2014), 655-687.

[7] I.E. Colak, J. Llibre AND C. VAlls, Bifurcation diagrams for Hamiltonian linear type centers of linear plus cubic homogeneous polynomial vector fields, J. of Differential Equations 258 (2015), 846-879.

[8] I.E. Colak, J. Llibre AND C. VAlls, Bifurcation diagrams for Hamiltonian nilpotent centers of linear plus cubic homogeneous polynomial vector fields, J. Differential Equations 262 (2017), 5518-5533.

[9] C. Christopher, J. Llibre, C. Pantazi And X. Zhang, Darboux integrability and invariant algebraic curves for planar polynomial systems, J. of Physics A: Math.and Gen. 35 (2002), 2457-2476.

[10] H. Dulac, Détermination et integration d'une certaine classe d'équations différentielle ayant par point singulier un centre, Bull. Sci. Math. Sér. (2) 32 (1908), 230-252.

[11] F. Dumortier, J. Llibre And J.C. ArtÉs, Qualitative theory of planar differential systems, Universitext, Springer-Verlag, 2006.

[12] W. Fulton, Algebraic Curves, Mathematics Lecture Note Series, W.A. Benjamin, 1974.

[13] P. Griffiths AND J. Harris, Principles of algebraic geometry, Wiley-Interscience, New York, 1978.

[14] D. Hilbert, Mathematische Probleme, Lecture, Second Internat. Congr. Math. (Paris, 1900), Nachr. Ges. Wiss. G"ottingen Math. Phys. KL. (1900), 253-297; English transl., Bull. Amer. Math. Soc. 8 (1902), 437-479; Bull. (New Series) Amer. Math. Soc. 37 (2000), 407-436.

[15] Yu. Ilyashenko, Centennial history of Hilbert's 16th problem, Bull. (New Series) Amer. Math. Soc. 39 (2002), 301-354.

[16] W. KAPTEYN, On the midpoints of integral curves of differential equations of the first degree, Nederl. Akad. Wetensch. Verslag. Afd. Natuurk. Konikl. Nederland (1911), 1446-1457 (Dutch).

[17] W. KAPTEYn, New investigations on the midpoints of integrals of differential equations of the first degree, Nederl. Akad. Wetensch. Verslag Afd. Natuurk. 20 (1912), 1354-1365; 21, 27-33 (Dutch).

[18] R.E. KooiJ And C. Christopher, Algebraic invariant curves and the integrability of the polynomial systems, Appl. Math. Lett 6 (No. 4) (1993), 51-53.

[19] J. LI, Hilbert's 16th problem and bifurcations of planar polynomial vector fields, Internat. J. Bifur. Chaos Appl. Sci. Engrg. 13 (2003), 47-106.

[20] J. Llibre AND D. XIAO, On the configurations of centers of planar Hamiltonian Kolmogorov cubic polynomial differential systems, preprint, 2017.

[21] K.E. MaLkin, Criteria for the center for a certain differential equation, (Russian) Volz. Mat. Sb. Vyp. 2 (1964), 87-91.

[22] I. PALAsti, The maximal number of quadrilaterals bounded by general straight lines in a plane, Periodica Mathematica Hungarica 6(4)(1975), 323-341.

[23] H. Poincaré, Mémoire sur les courbes définies par une équation differentielle, J. Maths. Pures Appl. 7 1881, 375-422.

[24] D. Schlomiuk, Algebraic particular integrals, integrability and the problem of the center, Trans. Amer. Math. Soc. 338 (1993), 799-841. 
[25] N. I. VulPE, Affine-invariant conditions for the topological discrimination of quadratic systems with a center, Differential Equations 19 (1983), 273-280.

[26] N.I. Vulpe AND K.S. SibirskiI, Centro-affine invariant conditions for the existence of a center of a differential system with cubic nonlinearities, (Russian) Dokl. Akad. Nauk sssR 301 (1988), 1297-1301; translation in Soviet Math. Dokl. 38 (1989), 198-201

[27] Z. Zhang, T. Ding, W. Huang And Z. Dong, Qualitative Theory of Differential Equations, Translations of Mathematical Monographs 101, Amer. Math. Soc., Providence, 1991.

[28] H. ŻoŁA̧DEK, Quadratic systems with center and their perturbations, J. Differential Equations 109 (1994), 223-273.

[29] H. ŻoŁA̧DEK, On a certain generalization of Bautin's theorem, Nonlinearity 7 (1994), 273279

[30] H. ŻOŁA̧DEK, The classification of reversible cubic systems with center, Topol. Methods Nonlinear Anal. 4 (1994), 79-136.

[31] H. ŻoŁA̧DEK, Remarks on: "The classification of reversible cubic systems with center, Topol. Methods Nonlinear Anal. 4 (1994), 79-136", Topol. Methods Nonlinear Anal. 8 (1996), 335342.

Departament de Matemátiques, Universitat Autónoma de Barcelona, 08193 Bellaterra, Barcelona, Catalonia, Spain

E-mail address: jllibre@mat.uab.cat 